\title{
A new Training Algorithm for Neuro-Fuzzy Networks
}

\author{
Stefan Jakubek ${ }^{1}$ and Nikolaus Keuth ${ }^{2}$ \\ 1 Vienna University of Technology, \\ Institute for Mechanics and Mechatronics, \\ Gußhausstr. 27-29/E325, A-1040 Vienna, Austria \\ jakubek@impa.tuwien.ac.at \\ http://www.impa.tuwien.ac.at \\ 2 AVL List GmbH, Hans-List Platz 1, A-8020 Graz, Austria, \\ Nikolaus.Keuth@avl.com
}

\begin{abstract}
In this paper a new iterative construction algorithm for local model networks is presented. The algorithm is focussed on building models with sparsely distributed data as they occur in engine optimization processes. The validity function of each local model is fitted to the available data using statistical criteria along with regularisation and thus allowing an arbitrary orientation and extent in the input space. Local models are consecutively placed into those regions of the input space where the model error is still large thus guaranteeing maximal improvement through each new local model. The orientation and extent of each validity function is also adapted to the available training data such that the determination of the local regression parameters is a well posed problem. The regularisation of the model can be controlled in a distinct manner using only two user-defined parameters. Examples from an industrial problems illustrate the efficiency of the proposed algorithm.
\end{abstract}

\section{Introduction}

Modeling and identification of nonlinear systems is challenging because nonlinear processes are unique in the sense that they may have an infinite structural variety compared to linear systems. A major requirement for a nonlinear system modeling algorithm is therefore universalness in the sense that a wide class of structurally different systems can be described.

The architecture of local model networks is capable of fulfilling these requirements and can therefore be applied to tasks where a high degree of flexibility is required. The basic principles of this modeling approach have been more or less independently developed in different disciplines like neural networks, fuzzy logic, statistics and artificial intelligence with different names such as local model networks, Takagi-Sugeno fuzzy models or neuro-fuzzy models $[3,9,2,12,10]$.

Local model networks possess a good interpretability. They interpolate local models, each valid in different operating regions, determined by so-called validity functions.

Many developments are focused on the bottleneck of the local model network which is the determination of these subdomains or validity functions, respectively.

One important approach is Fuzzy clustering as presented in $[4,3,14,6]$. An important issue in this field is the interpretability of the validity functions, for example as operating regimes. Recent developments can be found for example in [1,13].

Another development is the local linear model tree, LOLIMOT [11]. It is based on the idea to approximate a nonlinear map with piece-wise linear local models. The algorithm is designed such that it systematically bisects partitions of the input space. Local models that do not fit sufficiently well are thus replaced by two or more smaller 
models in the expectation that they will fit the nonlinear target function better in their region of validity.

In the practical application that motivated this work the amount of data available for identification is limited and the distribution of the data in their input space is sparse [5]. This imposes a limit on the network construction algorithm and it can lead to the situation that many local models are built where much fewer would be sufficient. Also, the sparseness of the input data gives rise to a more or less automated regularization that can be handled even by inexperienced users. The local model network construction algorithm presented in this paper can be seen as a mixture of classical fuzzy clustering techniques and the LOLIMOT construction algorithm. The present clustering algorithm takes into account the spatial distribution of the data in the input space and the prospective shape of the target function. The extent of the local model in the input space and its orientation are determined such that maximal statistical consistency/compliance with the sample data is achieved along with a spatial distribution of the data that yields a well conditioned problem.

The efficiency of the training algorithm is thus significantly increased resulting in less computational effort and fewer local models.

\section{Algorithm Description}

Fig. 1 illustrates the architecture of a local model network. Each local model (denoted as $L M_{i}$ ) takes the input vector $\boldsymbol{u}=\left[\begin{array}{llll}u_{1} & u_{2} & \ldots & u_{q}\end{array}\right]^{T}$ to compute its associated validity function $\Phi_{i}$ and its local estimation $\hat{y}_{i}$ of the nonlinear target function $f(\boldsymbol{u})$. The aggregate network output is the sum of all local model outputs $\hat{y}_{i}$ :

$$
\hat{y}(\boldsymbol{u})=\sum_{i=1}^{m} \Phi_{i}(\boldsymbol{u}) \hat{y}_{i}\left(\boldsymbol{u}, \boldsymbol{\theta}_{i}\right)
$$

Here, $\boldsymbol{\theta}_{i}$ is a vector containing the parameters of the local model and the local model output is generated from $\boldsymbol{u}$ and $\boldsymbol{\theta}_{i}$.

The structure of the validity function $\Phi_{i}$ was chosen as

$$
\Phi_{i}(\boldsymbol{u})=\exp \left(-\left[\left(\boldsymbol{u}-\boldsymbol{z}_{i}\right)^{T} \boldsymbol{A}_{i}\left(\boldsymbol{u}-\boldsymbol{z}_{i}\right)\right]^{\kappa_{i}}\right) \cdot \prod_{k=i+1}^{m}\left(1-\Phi_{k}(\boldsymbol{u})\right)
$$

The vector $\boldsymbol{z}_{i}$ contains the location of the current local model center, $\boldsymbol{A}_{i}$ is a symmetric and positive definite matrix that determines the orientation and extent of the validity function in the input space and the exponent $\kappa_{i}$ is a shape factor that determines the flatness of the validity function and thus also the degree of overlap between different local models. In the given examples this factor was set to 4 , a further discussion on the choice of $\kappa$ follows.

The structure of eq. (2) shows that every validity function is initally an exponential function with ellipsoidal contour lines. The product in (2) clips all subsequent validity functions from this original function, see Fig. 2. This choice has the following advantage: All subsequent local models are set into domains of the input space, where the preexisting model is insufficient. Eq. (2) ensures the dominance of the new local models in such domains. In Fig. 2 the situation is illustrated for a two-dimensional input space where a small domain is clipped from an originally ellipsoidal validity function.

The structure of (2) by iteself does not guarantee that validity functions yield a partition of unity. For that purpose the validity functions are normalized which is not explicitly oulined here for the sake of brevity. 


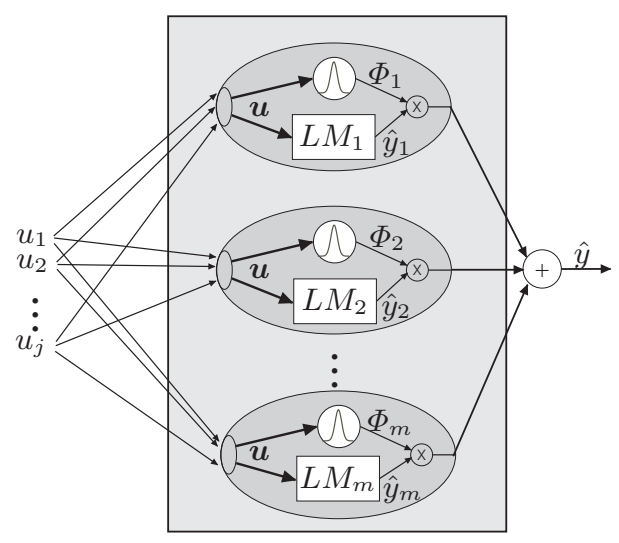

Fig. 1. Concept of local model networks
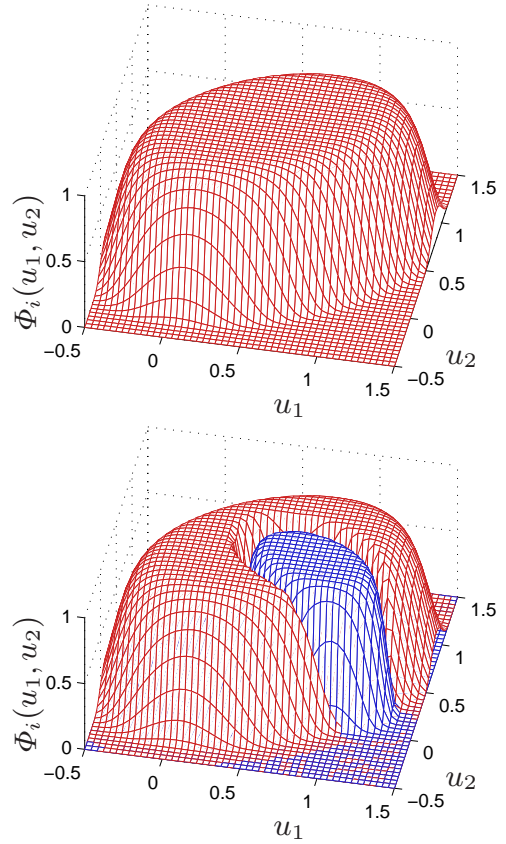

Fig. 2. Shape and hierarchy of validity functions

The overall quality of the network output is assessed either by the $R^{2}$ statistics or by the $R_{\text {pred }}^{2}$ statistics as outlined in [7]. The $R_{\text {pred }}^{2}$ statistics inherently describes the generalization quality of the model.

\subsection{Training procedure}

The local model network construction algorithm consists of an outer loop that determines the location, extent and orientation of each local model and a nested inner loop that optimises the parameters of the local model:

1. Start with an initial model: The initial model can either be a global model that covers all the available data or an "ordinary" local model as described below.

2. Set the next local model: Find the data point with the worst output error or prediction error. Choose the location of this data point as a candidate for the next local model. Design the orientation and extent of the new model such that it meets statistical compliance criteria with the sample data in the region of its validity. This procedure will be described in detail in the next section.

3. Compute the local model parameters and the associated statistical parameters that are necessary for the computation of prediction intervals

4. Test for Convergence: The performance criteria $R^{2}$ and $R_{p r e d}^{2}$ for the network output are computed. Once they have reached or exceeded their termination values the algorithm stops. Also, if no further improvement can be achieved the tree construction is stopped. Otherwise the algorithm proceeds with step 2. Especially in the case when the training data are subject to strong noise disturbance it is important that the training algorithm is controlled by a useful regularisation factor. Otherwise, the construction algorithm would create smaller and even smaller local models to improve the overall fit. In our case this is prevented by means of confidence levels as will be described in detail in the next section. 
After the local model network construction algorithm has finished to place local models each model undergoes a final examination for its contribution to the overall network output. If it turns out that a certain model does not contribute significantly anymore because it has been replaced by other models to a great extent it is removed.

\section{Local Model Design}

\subsection{Local Model Structure}

Every local model roughly consists of two parts: Its validity function $\Phi_{i}(\boldsymbol{u})$ and its model parameters $\boldsymbol{\theta}_{i}$. The output $\hat{y}_{i}$ of a local model at a point $\boldsymbol{u}$ in the input space is chosen as

$$
\hat{y}_{i}(\boldsymbol{u})=\boldsymbol{x}^{T}(\boldsymbol{u}) \cdot \boldsymbol{\theta}_{i} .
$$

Here, $\boldsymbol{x}^{T}(\cdot)$ represents a row vector of regressor functions which can be chosen arbitrarily. The advantage of the structure of (3) lies in the fact that $\hat{y}_{i}$ depends linearly on the parameters $\boldsymbol{\theta}_{i}$. Therefore, least-squares techniques can be applied for their computation. It remains to determine suitable regressor functions for $\boldsymbol{x}^{T}(\cdot)$ which will be outlined in section 3.3.

\subsection{Determination of the validity function}

In the presence of noise it is desirable that every local model should have optimal statistical significance. The algorithm presented in the sequel takes into account both the spatial distribution of the data and the expected shape of the target function and how well it can be modeled by the given regressors. In practical situations both the effect of noise and the prospective shape of the target function are unknown and the presented clustering approach turned out to be a reliable compromise.

1. The selection of a center "candidate" $z_{i}$ of a new local model is based on the estimation error $e_{j}=y_{j}-\hat{y}\left(\boldsymbol{u}_{j}\right)$. Here $\hat{y}\left(\boldsymbol{u}_{j}\right)$ is the model output from the network at it's current state when $i-1$ models have already been built:

$$
\hat{y}\left(\boldsymbol{u}_{j}\right)=\sum_{k=1}^{i-1} \Phi_{k}\left(\boldsymbol{u}_{j}\right) \hat{y}_{k}\left(\boldsymbol{u}_{j}, \boldsymbol{\theta}_{k}\right)
$$

Given $n$ data records $\boldsymbol{u}_{j}$ for model training the new center candidate is chosen as the data record where the output error is maximal:

$$
\boldsymbol{z}_{i, \text { cand }}=\boldsymbol{u}_{j_{m}} \text { with } j_{m}=\arg \max \left\|e_{j}\right\|
$$

This selection ensures that a new local model is set in the area where it is needed most.

2. Next, an initial set of neighbouring training data is chosen to compute an "initial" regression model. The minimum amount of data necessary for this initial model is determined by the requirement that the regression matrix must have maximum rank which depends both on the regressor functions and on the spatial distribution of the data. Next, the model statistics of the regression model are computed (see sec. 3.4) and a check is performed whether the initial data set lies within a prescribed prediction interval corresponding to a confidence level $\alpha$. If this is not the case the iteration terminates and the algorithm proceeds with the computation of the parameters of the validity function. Typically, the confidence level $\alpha$ is chosen between $90 \%$ and $99.9 \%$. 
3. Otherwise, further training data points in the vicinity of $\boldsymbol{z}_{i, \text { cand }}$ are added successively and the initial regression model together with the model statistics are adapted to these data using recursive least squares techniques. This step is repeated as long as $\alpha \%$ of the selected training data lie within the prediction interval. Thus it is ensured that the local model size and shape corresponds to the shape of the target function and the noisyness of the data.

4. Once training data have been selected in this way their distribution in the input space is used to determine the actual center along with the shape and extent of the validity function.

Figures 3 and 4 give an example for a nonlinear map $f(u): \mathbb{R} \rightarrow \mathbb{R}$ and $\alpha=97 \%$. The training data are represented by dots. Figure 3 depicts an "initial" regression model: The center candidate was chosen as $z_{i, c a n d}=0$ for this illustrative example, all data points in the interval $u \in[0 ; 0.17]$ were selected for the regression. The dashed curves represent the boundaries of the $97 \%$ prediction interval. Figure 4 depicts the "final" situation: Additional training data points had been added until finally about $97 \%$ of the selected data lie within the boundaries of the $97 \%$ confidence interval. The resulting validity function which was added to the figure for clarity extends from 0.1 to 0.8 having its maximum at 0.4 . Figure 5 illustrates the final situation for $\alpha=99.99 \%$. The increased confidence level $\alpha$ results in larger confidence intervals and consequently the validity function now has a greater extent. It can also be seen that in this example the shape of the nonlinearity has a much greater influence on the model size than in Figure 4. Figure 6 contains the same map $f(x)$, however, the influence of noise was drastically reduced. Altough the confidence level is still at $99.99 \%$ the extent of the validity function is now significantly smaller. From these examples it becomes obvious that the confidence level $\alpha$ serves as an excellent regularisation parameter that automatically adapts the local models to the noise corruption of the training data and to the regressor functions.

It remains to determine the nonlinear parameters of the validity function from the training data selected in the manner described above. Let $\boldsymbol{U}_{\text {sel }}$ be a matrix containing all these data records including the center candidate $\boldsymbol{z}_{i}$, i.e. every row vector of $\boldsymbol{U}_{\text {sel }}$ contains the coordinate vector $\boldsymbol{u}_{i}$ of a selected data point.

Then, the actual center is obtained by taking the center of gravity of the data in $U_{s e l}$ :

$$
\boldsymbol{z}_{i}=\operatorname{mean}\left(\boldsymbol{U}_{s e l}\right)
$$

where mean $(\cdot)$ takes the mean value over every column of its argument. The actual center is thus not the initial center candidate but the center of gravity of all chosen data points. This has the advantage that the algorithm is less sensitive to outliers in the data set.

The matrix $\boldsymbol{A}_{i}$ is computed by

$$
\boldsymbol{A}_{i}=\gamma \cdot\left[\operatorname{cov}\left(\boldsymbol{U}_{\text {sel }}\right)\right]^{-1}
$$

where $\operatorname{cov}(\cdot)$ denotes the empirical covariance matrix. This approach is taken from [8] where it was applied for the design of ellipsoidal basis function networks. The principal orientation of the new validity function is thus determined by $\left[\operatorname{cov}\left(\boldsymbol{U}_{\text {sel }}\right)\right]^{-1}$ and its extent in the input space is controlled by $\gamma$. The latter is chosen such that $\Phi_{i}$ is still 0.9 at the data point in $\boldsymbol{U}_{\text {sel }}$ which is located most remote from the new center $\boldsymbol{z}_{i}$. Thus, it is ensured that there are actually enough data points available for parameter estimation. Besides $\alpha$, both $\gamma$ and the shape parameter $\kappa$ constitute a second means of regularisation for the algorithm. The larger $\kappa$ is chosen the smaller the overlap between single local 


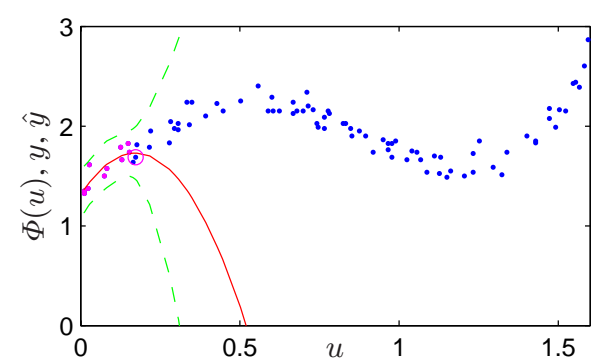

Fig. 3. Initial model with $\alpha=97 \%$

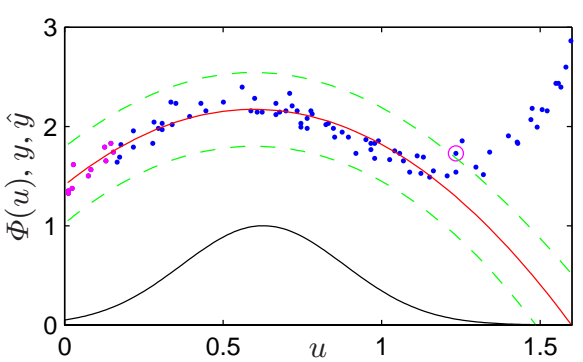

Fig. 5. Final local model with $\alpha=99.99 \%$

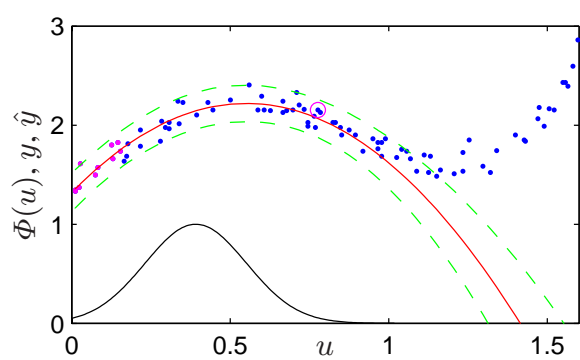

Fig. 4. Final local model with $\alpha=97 \%$

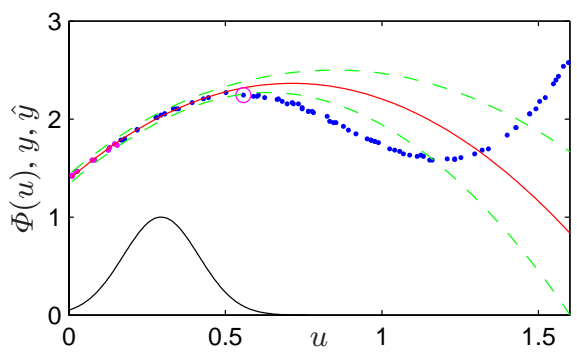

Fig. 6. Final local model with $\alpha=99.99 \%$ and less noise

models will be. Thus $\kappa$ directly controls the locality of the models. This does play an important role in the determination of covariances as will be outlined later. The factor $\gamma$ is directly related to the choice of $\kappa$ and just has to ensure the LS-problem is well posed as stated earlier. Consequently, the confidence level $\alpha$ and $\kappa$ are used to tune the training algorithm.

\subsection{Computation of the local model parameters}

As already mentioned earlier the output of each local model is computed through the regression model (3). It remains to determine suitable regression functions $\boldsymbol{x}^{T}(\boldsymbol{u})$. In general, there is no "optimal" solution that suits all possible nonlinear problems. The LOLIMOT-Algorithm [11] features linear models and in the present application the regressor functions were chosen as quadratic polynomials.

Let $\boldsymbol{X}$ denote a matrix constructed from the regression vectors of all $n$ training data records:

$$
\boldsymbol{X}=\left(\begin{array}{c}
\boldsymbol{x}^{T}\left(\boldsymbol{u}_{1}\right) \\
\boldsymbol{x}^{T}\left(\boldsymbol{u}_{2}\right) \\
\vdots \\
\boldsymbol{x}^{T}\left(\boldsymbol{u}_{n}\right)
\end{array}\right) \quad \text { and } \quad \boldsymbol{Q}_{i}=\left(\begin{array}{cccc}
\Phi_{i}\left(\boldsymbol{u}_{1}\right) & 0 & \cdots & 0 \\
0 & \Phi_{i}\left(\boldsymbol{u}_{2}\right) & \cdots & 0 \\
\vdots & \vdots & \ddots & \vdots \\
0 & 0 & \cdots & \Phi_{i}\left(\boldsymbol{u}_{n}\right)
\end{array}\right)
$$

where $\boldsymbol{Q}_{i}$ denotes a diagonal matrix composed from the $\Phi_{i}$, evaluated at the training data points. If $\boldsymbol{y}$ is a vector containing the values of the target function at the data points then the $i$-th local parameter vector is given by

$$
\boldsymbol{\theta}_{i}=\left(\boldsymbol{X}^{T} \boldsymbol{Q}_{i} \boldsymbol{X}\right)^{-1} \boldsymbol{X}^{T} \boldsymbol{Q}_{i} \boldsymbol{y}
$$




\subsection{Local Model Statistics}

Using the abbreviation

$$
\Theta_{Q}=\left(\boldsymbol{X}^{T} \boldsymbol{Q}_{j} \boldsymbol{X}\right)^{-1}
$$

the parameter variance $\operatorname{cov}(\boldsymbol{\theta})$ can be expressed by

$$
\operatorname{cov}(\boldsymbol{\theta})=\sigma_{n}^{2} \boldsymbol{\Theta}_{\boldsymbol{Q}} \boldsymbol{X}^{T} \boldsymbol{Q}_{\boldsymbol{i}}^{\mathbf{2}} \boldsymbol{X} \boldsymbol{\Theta}_{\boldsymbol{Q}}
$$

with the assumption that the measurement noise at different training data points is uncorrelated $E\left\{\boldsymbol{e} \boldsymbol{e}^{T}\right\}=\sigma_{n}^{2} \boldsymbol{I}$. It has to be mentioned that the noise covariance $\sigma_{n}^{2}$ is not known in most cases and therefore has to be replaced by the empirical covariance computed from the available data.

The variance of the local model output $\hat{y}_{i}\left(\boldsymbol{u}_{i}\right)$ is then calculated by

$$
\operatorname{cov}\left(\hat{y}_{i}(\boldsymbol{u})\right)=E\left\{\left(y_{i}-\hat{y}_{i}\right)^{2}\right\}==\sigma_{n}^{2}\left[\mathbf{1}+\boldsymbol{x}\left(\boldsymbol{u}_{i}\right) \boldsymbol{\Theta}_{\boldsymbol{Q}} \boldsymbol{X}^{T} \boldsymbol{Q}_{i}^{2} \boldsymbol{X} \boldsymbol{\Theta}_{\boldsymbol{Q}} \boldsymbol{x}^{T}\left(\boldsymbol{u}_{i}\right)\right]
$$

The prediction interval at a point $\boldsymbol{u}$ in the input space with a significance level of $\alpha$ is given by

$$
|\hat{y}(\boldsymbol{u})-y(\boldsymbol{u})|_{\alpha}=\sigma_{n} \sqrt{\mathbf{1}+\boldsymbol{x}(u) \boldsymbol{\Theta}_{\boldsymbol{Q}} \boldsymbol{X}^{T} \boldsymbol{Q}^{\mathbf{2}}{ }_{j} \boldsymbol{X} \boldsymbol{\Theta}_{\boldsymbol{Q}} \boldsymbol{x}(u)^{T}} \times t_{1-\frac{\alpha}{2}}
$$

As indicated, the $t$-statistics have to be computed for a significance level of $\alpha$ - $\%$ with the degrees of freedom depending on both the number of training data involved and the number of regressors.

\section{Global Model Design}

Apart from the local model design the interaction of all local models has to be considered in a global design. This involves the aggregation of the local model outputs as well as global model statistics, global prediction intervals and efficient ways to compute the $R^{2}$ and $R_{\text {pred }}^{2}$ statistics.

\subsection{Global Model Output}

As defined in (1) the global model output is a weighted sum of all local outputs. The weights depend on the location $\boldsymbol{u}$ in the input space and are determined from the validity functions $\Phi$ :

$$
\hat{y}(\boldsymbol{u})=\sum_{i=1}^{m} \Phi_{i}(\boldsymbol{u}) \hat{y}_{i}\left(\boldsymbol{u}, \boldsymbol{\theta}_{i}\right)
$$

As already mentioned earlier the design parameters of the validity function, $\kappa$ in particular implicitly determine the degree of overlap between the local models and thus serve as a regularisation parameter. It is straightforward that strong overlap in general leads to smoother function approximation. 


\subsection{Global Prediction Intervals}

Computation of confidence intervals at the global level involves two steps:

1. Computation of the covariance

2. Determination of the effective degrees of freedom at the global level for the $t_{\alpha}$ statistics.

Step 1 is straightforward since the global model output is a linear combination of the local model as given by (1). Strictly speaking, the outputs of different local models $\hat{y}_{i}(\boldsymbol{u})$ are not independent since the underlying models all originate from the same data. However, the shape parameter $\kappa$ in (2) entails that there is only little overlap between single local models. The statistical interdependencies between local models is therefore neglegible, which was also verified in numerous tests.

$$
\operatorname{cov}(\hat{y}(\boldsymbol{u}))=\sum_{i=1}^{m} \Phi_{i}^{2}(\boldsymbol{u}) \operatorname{cov}\left(\hat{y}_{i}(\boldsymbol{u})\right)
$$

In the above formula $\operatorname{cov}\left(\hat{y}_{i}(\boldsymbol{u})\right)$ is taken from (12). It is noteworty that (14) does not contain $\sigma_{n}^{2}$ directly. The covariance $\operatorname{cov}\left(\hat{y}_{i}(\boldsymbol{u})\right)$ of every local model depends on the model structure and on the local measurement noise. This means that (14) could also be applied if the measurment noise varies from local model to local model, provided that $\operatorname{cov}\left(\hat{y}_{i}(\boldsymbol{u})\right)$ is computed correctly.

Step 2 is taken from [10] where the effective number of parameters $n_{\text {eff }}$ is determined from the trace of the so-called "smoothing matrix".

The remaining degrees of freedom then result to $D O F=n-n_{\text {eff }}$ and this is the basis for the computation of the $t$-statistics. It is noteworthy that $n_{e f f}$ is not necessarily an integer number. During the tree construction process it sometimes happens that the validity function of a certain local model is successively clipped away by subsequent models so that it finally doesn't contribute anymore to the model quality. In the present application these models are finally removed if their $n_{e f f, i}$ has dropped below 1 .

\section{Results}

In the given example measurement data from an IC-Engine are considered. For the development and testing of the presented algorithm measurement databases from different engine manufacturers were used. The dimension of the input space in these databases varies from three to eight.

The three-dimensional database will be illustrated in detail since in this case some demonstrative graphical illustrations can be given.

In particular, the air efficiency $A_{e}$ of a valvetronic engine shall be represented as a function of engine speed $n$, valve lift $L_{v}$ and intake closure time $T_{c}$ :

$$
A_{e}=f\left(n, L_{v}, T_{c}\right)
$$

The resulting network models the map $f: \mathbb{R}^{3} \rightarrow \mathbb{R}$ with an accuracy of $R^{2}=$ 0.9941 and $R_{\text {pred }}^{2}=0.9931$ using 7 local models. In comparison, the LOLIMOTalgorithm using the same quadratic local model structure needs 30 local models to reach the same accuracy.

Figures 7, 8 and 9 depict the network output each with one input kept constant. Apart from the network output the corresponding training data points are depicted and the validity functions are represented by contour lines. Note that not all seven validity 


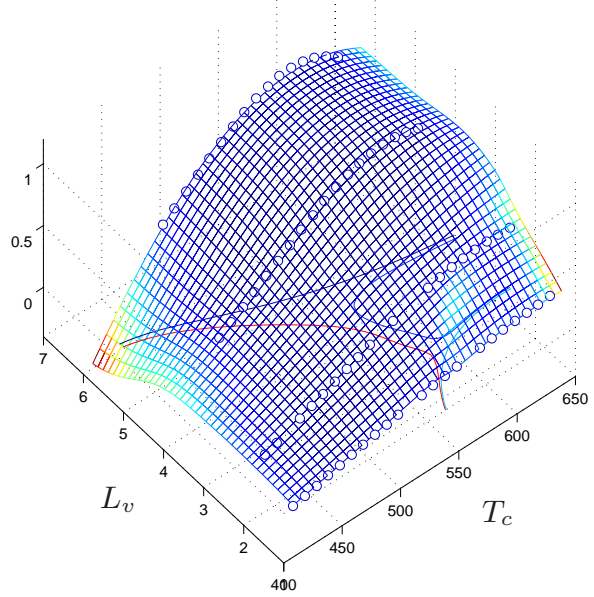

Fig. 7. Valvetronic engine example: Model output for $n=2600=$ const.

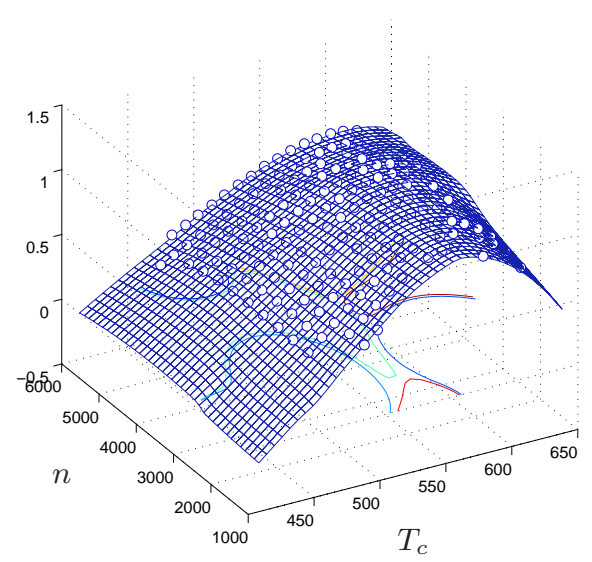

Fig. 9. Valvetronic engine example: Model output for $L_{v}=6=$ const.

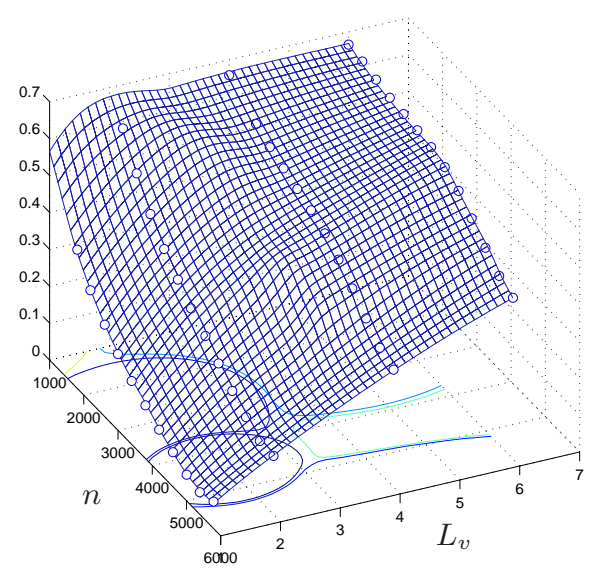

Fig. 8. Valvetronic engine example: Model output for $T_{c}=498=$ const.

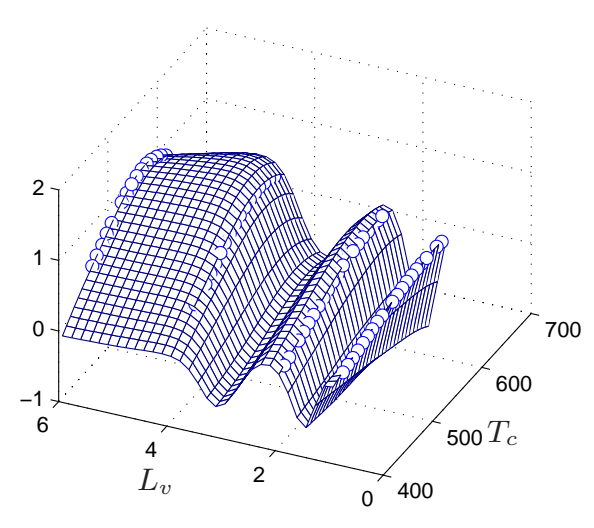

Fig. 10. Valvetronic engine example: Perceptron output for $n=2600=$ const.

functions are visible in the respective intersection planes. It can be seen that they are fitted to the shape of the target function in an efficient way.

The measurement data considered in this example were also used to compare the performance of the presented network architecture and training algorithm to that of a perceptron network. The number of hidden neurons was chosen such that the effective number of weights corresponds to the number of parameters in the competing local model network. The weights of the perceptron network were optimized using standard training algorithms in combination with different regularization techniques in order to balance bias and variance errors. In a Monte-Carlo simulation it turned out that despite this efforts the perceptron has particular difficulties interpolating the large gaps between the individual valve lift values (cf. Fig. 7 and Fig. 10).

Altogether, 300 Perceptrons were trained in the manner described above, resulting in the following statistical assessment: $13 \%$ of all Perceptrons had a performance superior to that of the local model network. They exceeded the $R_{\text {pred }}^{2}$ value of 0.9931 and the interpolation behaviour was better. $13 \%$ of all Perceptrons produced a comparable per- 
formance in terms of the same criteria. The remaining $74 \%$ of all 300 perceptrons were outperformed by the local model network, cf. Fig. 10. In many cases the confidence interval was unacceptably large for practical applications.

\section{Conclusion and Outlook}

In this paper a new iterative tree construction algorithm for local model trees was presented. The validity functions of the generated model are closely fitted to the available data by allowing an arbitrary orientation and extent of the validity function of each local model in the input space.

The regularisation of the model can be controlled by a shape factor $\kappa$ which determines the overlap between local models and by a confidence level $\alpha$ that controls the relative size of each local model.

The application to data from internal combustion engines shows that the proposed algorithm produces excellent results with a relatively low number of local models.

\section{References}

1. J. Abonyi, R. Babuska, and F. Szeifert. Modified gath-geva fuzzy clustering for identification of takagi-sugeno fuzzy models. In IEEE Tarnsactions on systems, man. and Cybernetics, volume 32, pages 612-621. IEEE, 2002.

2. R. Babuska. Recent Advances in Intelligent Paradigms and Applications. Springer-Verlag, Heidelberg, 2002.

3. R. Babuska and H.B. Verbruggen. An overview of fuzzy modeling for control. control Engineering Practice, 4(11):1593 - 1606, 1996.

4. J.C. Bezdek, E.C.-K. Tsao, and N.R. Pal. Fuzzy kohonen clustering networks. In IEEE International Conference on Fuzzy Systems, pages 1035 - 1043. IEEE, 1992.

5. A. Bittermann, E. Kranawetter, Krenn J., Ladein B., Ebner T., H. Altenstrasser, H. Koegeler, and K. Gschweitl. Emissionsauslegung des dieselmotorischen fahrzeugantriebs mittels doe und simulationsrechnung. Motorentechnische Zeitschrift, 2004.

6. I. Gath and A.B. Geva. Unsupervised optimal fuzzy clustering. IEEE Transactions on Pattern Analysis and Machine Intelligence, 11(7):773-781, 1989.

7. X. Hong, P.M. Sharkey, and K. Warwick. A robust nonlinear identification algorithm using press statistics and forward regression. In IEEE Transactions on Neural Networks, volume 14 , pages $454-458$. IEEE, 2003.

8. S. Jakubek and T. Strasser. Artificial neural networks for fault detection in large-scale data acquisition systems. Engineering Applications of Artificial Intelligence, 17:233-248, May 2004.

9. T. A. Johansen and B. A. Foss. Operating regime based process modeling and identification. Computers and Chemical Engineering, 21:159 - 176, 1997.

10. O. Nelles. Nonlinear System Identification. Springer Verlag, 1st edition, 2002.

11. O. Nelles and R. Isermann. Basis function networks for interpolation of local linear models. In IEEE Conference on Decision and Control (CDC), pages 470-475, 1996.

12. X.M. Ren, A.B. Rad, P.T. Chan, and L.L. Wai. Identification and control of continuous-time nonlinear systems via dynamic neural networks. Industrial Electronics, IEEE Transactions on, 50(3):478-486, June 2003.

13. A. Trabelsi, F. Lafont, M. Kamoun, and Enea G. Identification of nonlinear multivariable systems by adaptive fuzzy takagi-sugeno model. International Journal of Computational Cognition (http://www.YangSky.com/yangijcc.htm), 2(3):137-153, September 2004.

14. J. Yen and Wang L. Application of statistical information criteria for optimal fuzzy model construction. IEEE Transactions on Fuzzy Systems, 6(3):362 - 372, August 1998. 